\title{
Preserved antibody levels and loss of memory B cells against pneumococcus and tetanus after splenectomy: Tailoring better vaccination strategies
}

\author{
M. Manuela Rosado ${ }^{1}$, Francesco Gesualdo ${ }^{2}$, Valentina Marcellini ${ }^{1}$, \\ Antonio Di Sabatino ${ }^{3}$, Gino Roberto Corazza ${ }^{3}$, Maria Paola Smacchia ${ }^{4}$, \\ Bruno Nobili $^{5}$, Carlo Baronci ${ }^{6}$, Lidia Russo ${ }^{6}$, Francesca Rossi $^{5,6}$, \\ Rita De Vito ${ }^{7}$, Luciana Nicolosi $^{8}$, Alessandro Inserra ${ }^{9}$, Franco Locatelli ${ }^{6,10}$, \\ Alberto E. Tozzi ${ }^{2}$ and Rita Carsetti ${ }^{1,11}$ \\ ${ }^{1}$ Immunology Area, Bambino Gesù Children's Hospital IRCCS, Rome, Italy \\ 2 Multifactorial Diseases and Complex Phenotypes Area, Bambino Gesù Children's Hospital \\ IRCCS, Rome, Italy \\ ${ }^{3}$ Department of Medicine, University of Pavia, Policlinico San Matteo IRCCS, Pavia, Italy \\ ${ }^{4}$ Department of Pediatrics, Sapienza University of Rome, Rome, Italy \\ ${ }^{5}$ Department of Pediatrics, Second University of Naples, Naples, Italy \\ ${ }^{6}$ Department of Oncohematology, Bambino Gesù Children's Hospital IRCCS, Rome, Italy \\ ${ }^{7}$ Unit of Pathology, Bambino Gesù Children's Hospital IRCCS, Rome, Italy \\ ${ }^{8}$ Department of Pediatrics, Bambino Gesù Children's Hospital IRCCS, Rome, Italy \\ ${ }^{9}$ Division of Pediatric Surgery, Bambino Gesù Children's Hospital IRCCS, Rome, Italy \\ ${ }^{10}$ Department of Pediatrics, University of Pavia, Pavia, Italy \\ ${ }^{11}$ Department of Laboratories, Bambino Gesù Children's Hospital IRCCS, Rome, Italy
}

Splenectomized patients are exposed to an increased risk of septicemia caused by encapsulated bacteria. Defense against infection is ensured by preformed serum antibodies produced by long-lived plasma cells and by memory B cells that secrete immunoglobulin in response to specific antigenic stimuli. Studying a group of asplenic individuals (57 adults and 21 children) without additional immunologic defects, we found that spleen removal does not alter serum anti-pneumococcal polysaccharide (PnPS) IgG concentration, but reduces the number of PnPS-specific memory B cells, of both IgM and IgG isotypes. The number of specific memory B cells was low in splenectomized adults and children that had received the PnPS vaccine either before or after splenectomy. Seven children were given the 13-valent pneumococcal conjugated vaccine after splenectomy. In this group, the number of PnPS-specific IgG memory B cells was similar to that of eusplenic children, suggesting that pneumococcal conjugated vaccine administered after splenectomy is able to restore the pool of anti-PnPS IgG memory B cells. Our data further elucidate the crucial role of the spleen in the immunological response to infections caused by encapsulated bacteria and suggest that glycoconjugated vaccines may be the most suitable choice to generate IgG-mediated protection in these patients.

Keywords: Asplenia - Memory B cells · Serum anti-PnPS · Splenectomy · Streptococcus pneumoniae

Additional supporting information may be found in the online version of this article at the publisher's web-site

Correspondence: Dr. M. Manuela Rosado

e-mail:m.rosado@wildmail.com 


\section{Introduction}

Asplenic patients have an increased susceptibility to bacterial infections that can evolve into severe and often lethal overwhelming postsplenectomy infection (OPSI) [1,2]. Lifetime risk of developing an OPSI is nearly $1-5 \%$ and mortality rates range between 40 and $70 \%$ [3]. Streptococcus pneumoniae is the most common pathogen causing bacteremia in splenectomized patients, followed by Haemophilus influenzae, Neisseria meningitidis, Escherichia coli, Salmonella, Pseudomonas, and Klebsiella [3,4].

The red pulp of the spleen represents a very important defense from bacteremia. In the red pulp of the spleen, the blood flows slowly in a large net of sinusoids. Macrophages, located among endothelial cells, have the function of removing and destroying particulate antigens, such as bacteria [5-7]. In the absence of the spleen, bacteria can therefore accumulate and replicate in the blood causing septic shock.

Over the last few years, the role of the spleen in the maintenance of a pool of memory B cells involved in the protection against encapsulated bacteria has been also demonstrated. In particular, we showed that splenectomized patients lack IgM memory B cells [8]. This cell population, also known as marginal zone B cells or effector memory B cells, is produced by a T-independent mechanism [9] and inhabits the marginal zone of the spleen. IgM memory B cells generate the response to pneumococcal polysaccharide (PnPS) vaccines in vivo [10] and produce anti-pneumococcal antibodies in vitro [11]. IgM antibodies are indispensable for efficient opsonization and subsequent phagocytosis of encapsulated bacteria. The marginal zone also hosts switched memory B cells, able to secrete IgG antibodies, generated by T-dependent responses in the germinal centers $[12,13]$. Whereas IgM memory B cells are permanently depleted after splenectomy, switched memory cells can be regenerated by new immune responses in the lymph nodes and thus increase with time after splenectomy [8].

Preventive measures such as antibiotic prophylaxis and vaccination against encapsulated bacteria are recommended [14] for limiting the high burden of invasive bacterial infections in asplenic patients [15]. Until 2012, the Advisory Committee on Immunization Practices recommended immunization with PnPS vaccine in asplenic individuals at the time of diagnosis and a booster dose after 5 years [16]. In 2012, new guidelines have been produced, recommending the administration of 13 -valent pneumococcal conjugate vaccine (PCV13) in addition to the 23-valent PnPS vaccine in adults [17]. According to these guidelines, the PnPS vaccine should be administered after PCV13 to all asplenic children in order to protect them against the additional serotypes not included in PCV13 $[18,19]$.

In order to further investigate the consequences of splenectomy on the defense against $S$. pneumoniae, we studied a population of asplenic children and adults and compared them to age-matched controls. We only enrolled patients that had been splenectomized but had an otherwise intact immune system and were not being treated with immunosuppressive drugs to ensure that results were only due to the lack of the spleen, but not to other either primary or secondary alterations of the immune response.

We found that the concentration of serum antibodies against pneumococcal polysaccharides was not affected by removal of the spleen, but memory B cells able to produce anti-PnPS antibodies of both IgM and IgG isotypes were strongly reduced, despite the fact that most splenectomized adults and children had received polysaccharide vaccines.

\section{Results}

\section{Serum levels of IgG against S. pneumoniae are comparable in asplenic patients and in healthy donors}

The level of serum antibodies is considered the primary marker of protection against infections. We compared the serum concentration of IgG against PnPS in splenectomized children and adults with that of healthy controls. We found that the levels of antiPnPS IgG were similar in the two groups, independently of age and vaccination history (Fig. 1A and B).

We also measured the serum levels of anti-tetanus toxoid IgG and found that in asplenic children anti-Tx IgG concentration was increased as compared to healthy children (Fig. 1C). This difference was most probably caused by the fact that, being older compared to controls, the group of splenectomized children received more booster doses of ant-tetanus vaccine. In contrast, splenectomized adults showed lower anti-Tx IgG serum levels than controls (Fig. 1D).

According to our results asplenia does not affect the concentration of IgG antibodies against $S$. pneumoniae. Thus, we investigated other immunological defects caused by absence of the spleen that may explain susceptibility to pneumococcal infections.

\section{Loss of memory B cells specific for S. pneumoniae and tetanus in splenectomized adults}

The spleen contains $15-25 \%$ of the lymphocytes of our body [20]. With the removal of the spleen all B cells contained in the organ are lost. These include transitional B cells emigrated from the bone marrow, mature-naïve B cells that populate the lymphoid follicles and memory $\mathrm{B}$ cells that reside in the marginal zone (Supporting Information Fig. 1A-C) [21]. Transitional B cells are continuously de novo generated in the bone marrow and rapidly differentiate into mature naïve B cells. Reconstitution of memory B cells, representing our antigenic experience, requires a longer time because they can only be replaced by new immune responses.

In order to confirm that the anti-pneumococcal specificity is contained in the spleen, we obtained material from four patients (described in Supporting Information Table 1 and Supporting Information Fig. 1). We used $\mathrm{CpG}$ induced differentiation followed by ELISPOT as a tool to evaluate the function and specificity of memory B cells [22]. We measured the number of IgM- and 
A

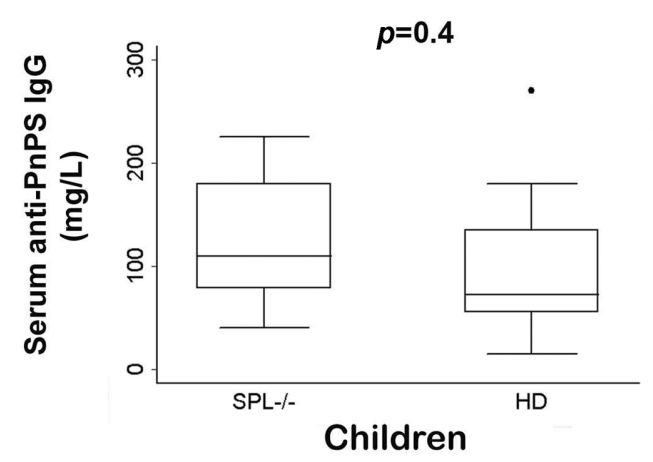

C

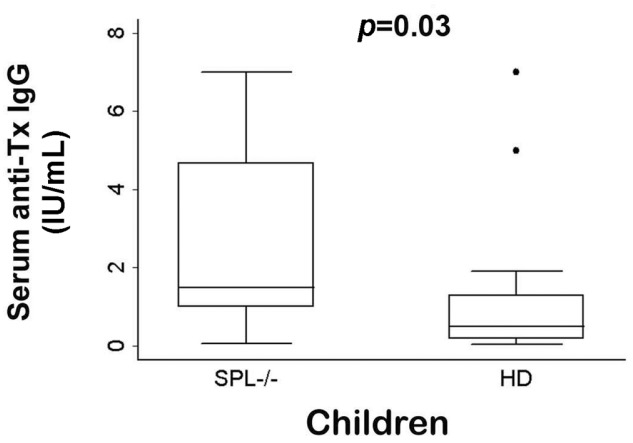

B

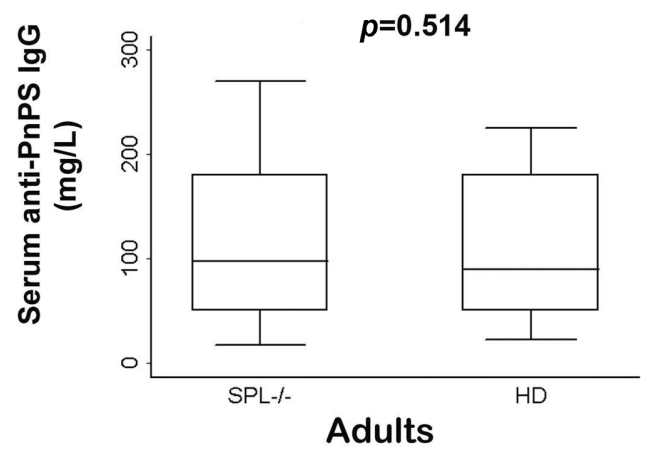

D

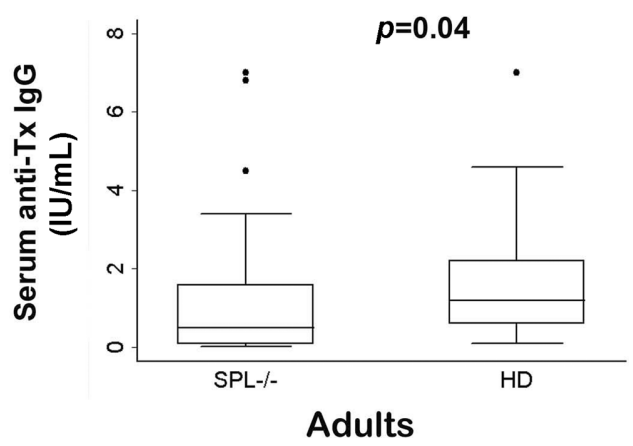

Figure 1. Anti-PnPS and $\alpha$-tetanus toxoid IgG serum concentrations in splenectomized and healthy controls. (A) The $\alpha$-PnPS IgG serum concentration $(\mathrm{mg} / \mathrm{L})$ in splenectomized (SPL-/-, $n=21)$ and control infants (HD, $n=19)$ and (B) in splenectomized adults $(n=57)$ versus controls $(n=47)$ are shown. (C) The $\alpha$-tetanus toxoid IgG serum concentration (IU/mL) in splenectomized (SPL-/-) and control infants (HD) and (D) in splenectomized adults versus controls are shown. Data are expressed as box-and-whisker plots representing the median (solid line), interquartile ranges (boxes), and minimum and maximum nonoutlier values (whiskers), respectively. Statistical significance was determined by the Mann-Whitney U test.

IgG-secreting cells. As demonstrated before [10], the number of total IgM-secreting cells was lower than that of total IgG-secreting ones (Supporting Information Fig. 1D). However, B cells producing antibodies with specificity for PnPS were mostly of IgM isotype. Eleven percent of the IgM-secreting memory B cells bound pneumococcal polysaccharides in ELISPOT confirming previous reports on the splenic localization of the anti-pneumococcal response [10]. In the IgG-secreting compartment (Supporting Information Fig. 1E), pneumococcus-specific cells were rare (0.4\%).

In order to investigate to what extent the loss of the splenic repository of memory B cells affects the circulating pool, we isolated peripheral blood mononuclear cells (PBMCs) from asplenic and healthy adults and stained them for CD19, CD27, IgM, and IgD (Supporting Information Fig. 2A). We measured the frequency of B cells $\left(\mathrm{CD} 19^{+}\right)$, total memory B cells $\left(\mathrm{CD} 19^{+} \mathrm{CD} 27^{+}\right), \mathrm{IgM}$ memory $\left(\mathrm{CD} 19^{+} \mathrm{CD}_{27}{ }^{+} \operatorname{IgM}^{+} \operatorname{IgD}^{+}\right)$, and switched memory B cells $\left(\mathrm{CD} 19^{+} \mathrm{CD}^{2} 7^{+} \mathrm{IgM}^{-} \mathrm{IgD}^{-}\right)$. Results are summarized in Supporting Information Table 2).

We found that in splenectomized adults the frequency of total $B$ cells varied among individuals, but was increased in comparison with that in healthy controls (Supporting Information Table 2 and Supporting Information Fig. 2B).

In contrast, memory B cells (Supporting Information Fig. 2C) of both switched and IgM memory isotype (Supporting
Information Fig. 2D and E) were always and significantly reduced. The median proportions of $\operatorname{IgM}(3.4 \%)$ and switched (9\%) memory cells were significantly lower than in healthy individuals $(16.1 \%$ for both cell populations, Supporting Information Table 2).

By ELISPOT we measured the number of antibody-secreting cells in response to $\mathrm{CpG}$, reflecting the number and function of memory B cells [11]. We observed that both IgM (Fig. 2A) and IgG-secreting cells (Fig. 2B) were significantly reduced confirming the phenotypic analysis.

In the peripheral IgM memory pool of asplenic adults, the anti-PnPS specificity was also significantly lower than in controls (Fig. 2C). The number of anti-PnPS IgG memory B cells was reduced to the same extent (Fig. 2D).

We also measured the frequency of memory B cells secreting antibodies directed toward a second antigen, namely tetanus toxoid, against which all individuals are vaccinated in infancy. Memory B cells against the tetanus toxoid, both of IgM and IgG isotypes, were also significantly diminished in asplenic adults (Fig. 2E and $\mathrm{F}$ ).

All asplenic adults enrolled in our study received only PnPS vaccination as a preventive measure against pneumococcal infection [4]. In this group of patients, notwithstanding the previous vaccination, the number of memory cells specific for 
A
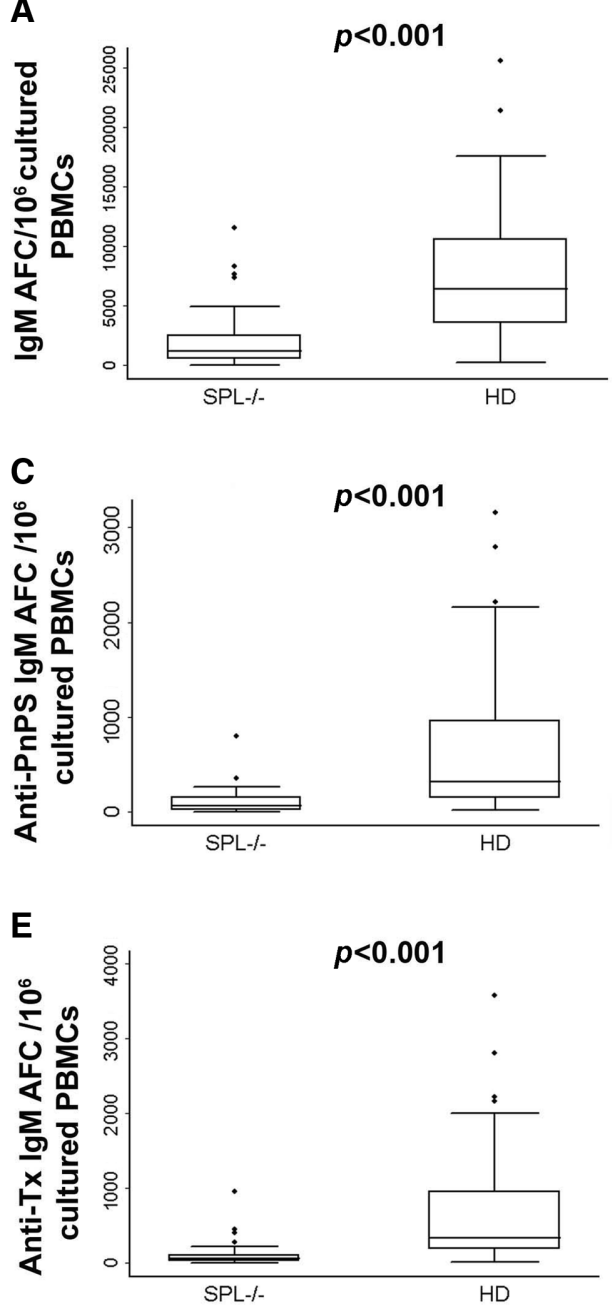

B
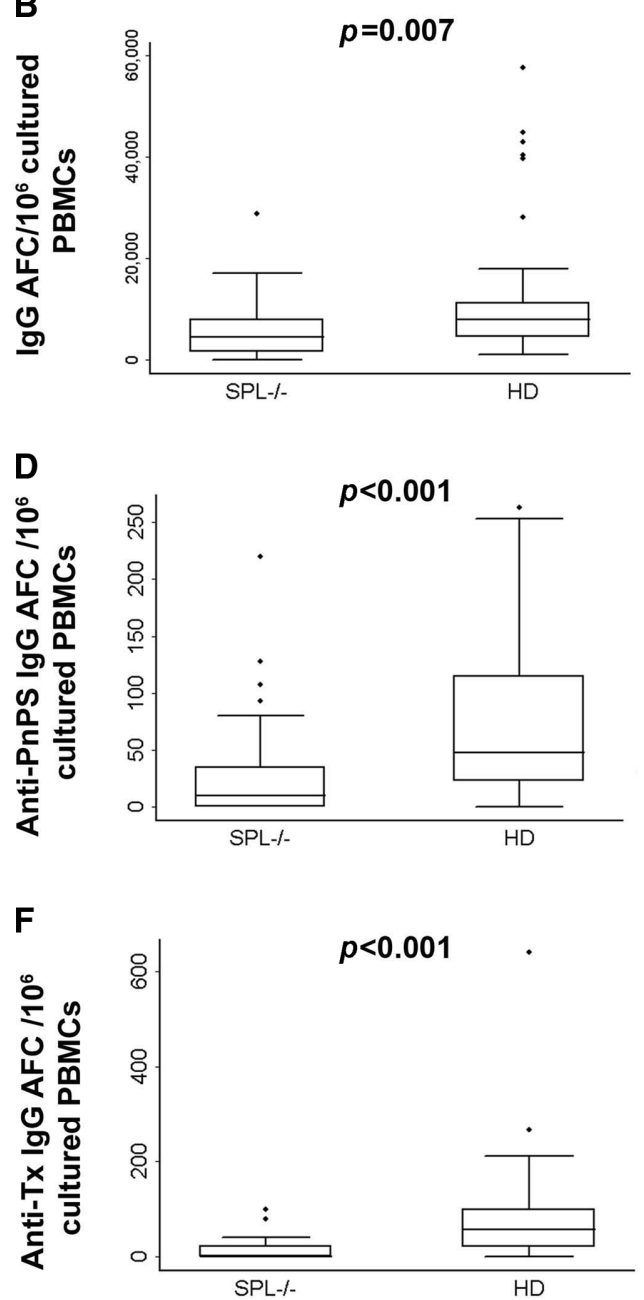

Figure 2. Number of memory B cells secreting IgM, IgG anti-PnPS IgM, and anti-PnPS IgG in the peripheral blood of splenectomized and healthy adults. PBMCs were stimulated with CPG for 5 days and the number of antibody-secreting cells was measured by ELISPOT. (A-F) The number of (A) total IgM, (B) total IgG, (C) anti-PnPS IgM, (D) anti-PnPS IgG, (E) anti-tetanus toxoid (Tx) IgM, and (F) anti-Tx IgG per million of total PBMCs in splenectomized and control adults are shown. Data are expressed as box-and-whiskers plots representing the median (solid line), interquartile ranges (boxes), and minimum and maximum nonoutlier values (whiskers) for 57 asplenic and 47 healthy adults. Statistical significance was determined by the Mann-Whitney U test.

pneumococcus was very low (Fig. 2C and D), indicating that PnPS vaccination does not generate long-term memory B cells in splenectomized individuals.

\section{Memory B cells in children}

We also analyzed peripheral B-cell subsets in asplenic children. Results are summarized in Supporting Information Table 3. We found that the frequency of total B cells was not significantly different from healthy controls of the same age (Fig. 3A), whereas the frequency of total memory and IgM memory B cells was diminished, confirming the important role of the spleen in the generation and maintenance of the IgM memory pool (Fig. 3B and C). The reduction of switched memory $B$ cells instead did not reach significance (Fig. 3D).
We used CpG induced differentiation followed by ELISPOT as a tool to evaluate memory B-cell specificity. We found that total IgM-secreting cells were severely reduced in asplenic children (Fig. 4A). IgG-secreting cells were also diminished (Fig. 4B). We also analyzed the number of IgM- and IgG-secreting cells with antipneumococcal and anti-tetanus specificity. In the peripheral blood of normal children, the frequency of B cells with anti-PnPS specificity was relatively high, accounting for $4 \%$ of all IgM-secreting cells, whereas specific IgG memory were rare $(0.2 \%$, see values for healthy donors in Figure 4C and D).

In asplenic children, anti-pneumococcal IgM-secreting cells were significantly lower than in healthy children (Fig. 4C), whereas IgG-secreting cells were reduced, but the difference did not reach significance (Fig. 4D).

We also measured the number of memory B cells specific for tetanus. Anti-tetanus IgM spots were reduced in asplenic children 
A

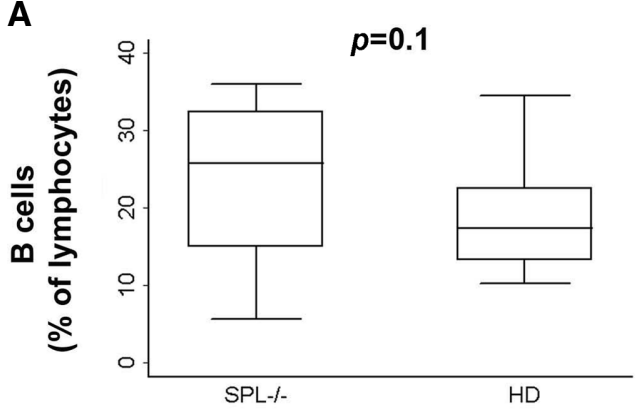

C

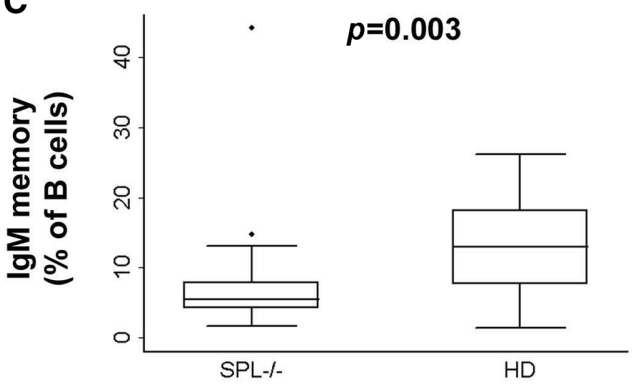

B
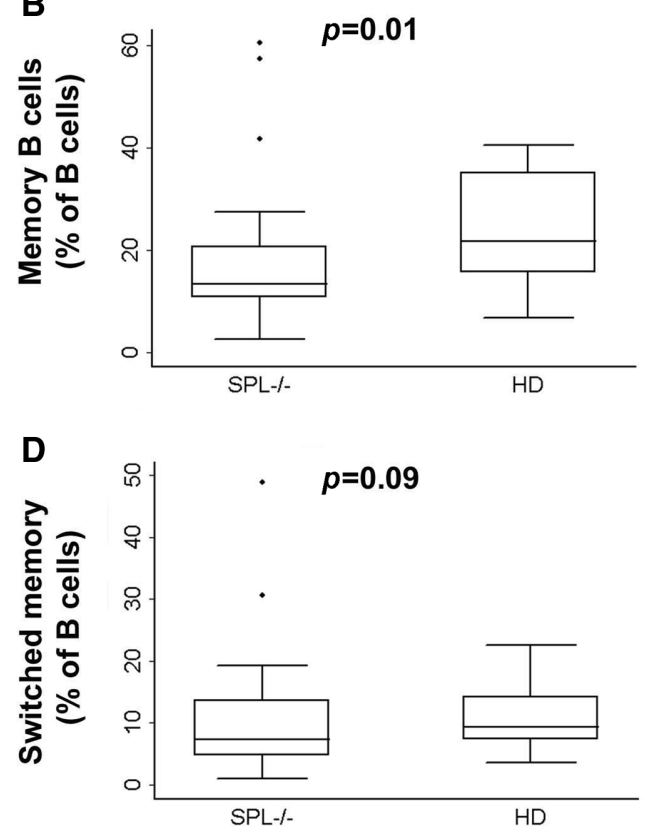

Figure 3. Peripheral blood B-cell subset frequencies in splenectomized children and healthy controls. PBMCs isolated from splenectomized and healthy children were stained for CD19, CD27, IgM, and IgD and analyzed by flow cytometry. (A-D) The frequency of (A) B cells (CD19+), (B) memory

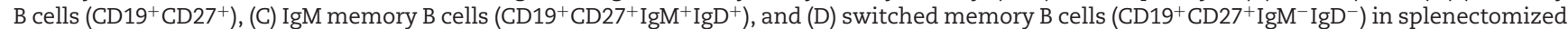
(SPL-/-) and healthy donors (HD) are shown. Data are expressed as box-and-whisker plots with median (solid line), interquartile ranges (boxes), and minimum and maximum nonoutlier values (whiskers) for 21 asplenic and 19 healthy children. Statistic differences between the two groups were determined by the Mann-Whitney U test.

(Fig. 4E), but anti-tetanus specific IgG secreting cells were in the normal range (Fig. 4F).

Thus, both adults and children have a marked reduction of IgM memory B cells with anti-PnPS and anti-tetanus specificity. These cells may produce natural antibodies, probably not effective against tetanus, but important in the defense against $S$. pneumoniae infections.

\section{The impact of vaccination on the memory B-cell pool}

Based on the observation that differences in the number of specific IgG memory B cells in adults are more evident than in children, we investigated whether recent immunization against $S$. pneumoniae in children may have affected our results. We have previously shown and now confirmed in both adults and children a reduction of IgM memory B cells, the B-cell population that responds to PnPS. Glycoconjugated vaccines are effective in infants, who physiologically lack IgM memory B cells, because protein-coupled polysaccharides generate a T-dependent response, resulting in a classical germinal center reaction that can take place in all lymphoid tissues, including the lymph-nodes draining the site of vaccine injection [23]. Thus, glycoconjugated vaccines do not require either the spleen or IgM memory B cells. According to current guidelines, at least one dose of this type of vaccine is recommended after splenectomy [17].
We correlated the frequency of IgG and IgM memory B cells and serum IgG specific for PnPS to the vaccination history of the children included in our study. In children that had received PnPS vaccination (before and/or after splenectomy) and in those that had been immunized with PCV before splenectomy, the number of specific IgG memory B cells was not increased (Fig. 5A). In contrast, we found that, in children that had received the glycoconjugated vaccine after splenectomy, the number of anti-PnPS IgG memory B cells was similar to that of eusplenic children (Fig. 5A). Serum concentration of anti-PnPS IgG increased in asplenic children vaccinated with PCV (Fig. 5B) after splenectomy, reflecting the increase of anti-PnPS specific IgG memory B cells in the same individuals.

Anti-pneumococcal IgM specific B cells were only marginally increased by the PCV vaccination (Fig. 5C).

\section{Discussion}

Prevention of pneumococcal disease in splenectomized/asplenic children and adults is a medical priority. A thorough investigation to optimize the use of the polysaccharide and/or the conjugated vaccine in asplenic patients is still lacking. In fact, guidelines often include asplenic patients in a broader category of immunodeficient and immunosuppressed individuals at higher risk of pneumococcal infections $[17,19]$. The immunological correlate most frequently 
A

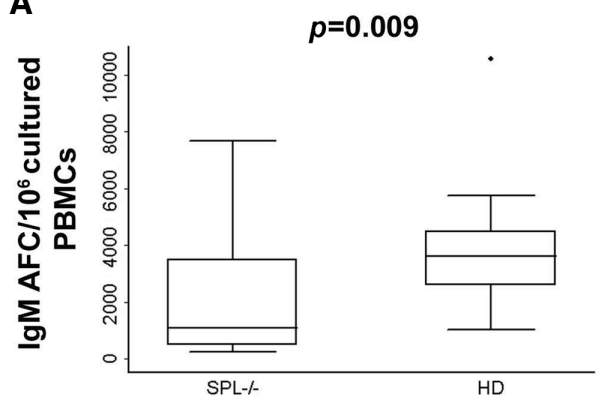

C

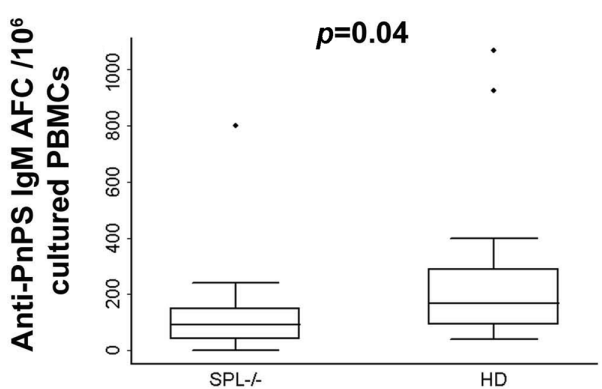

E

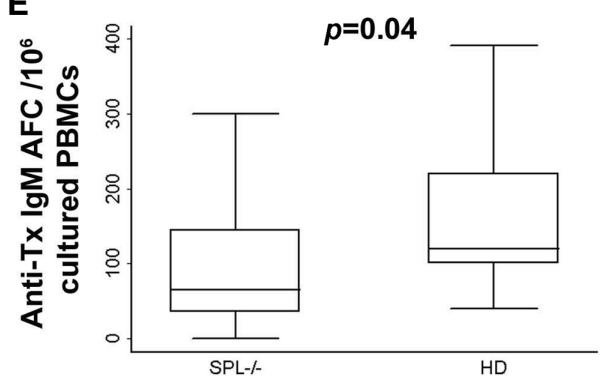

B

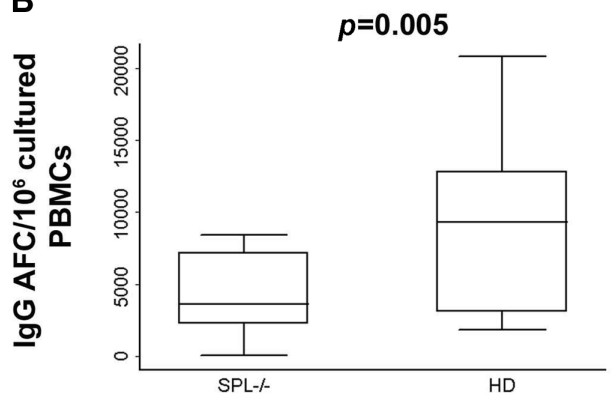

D
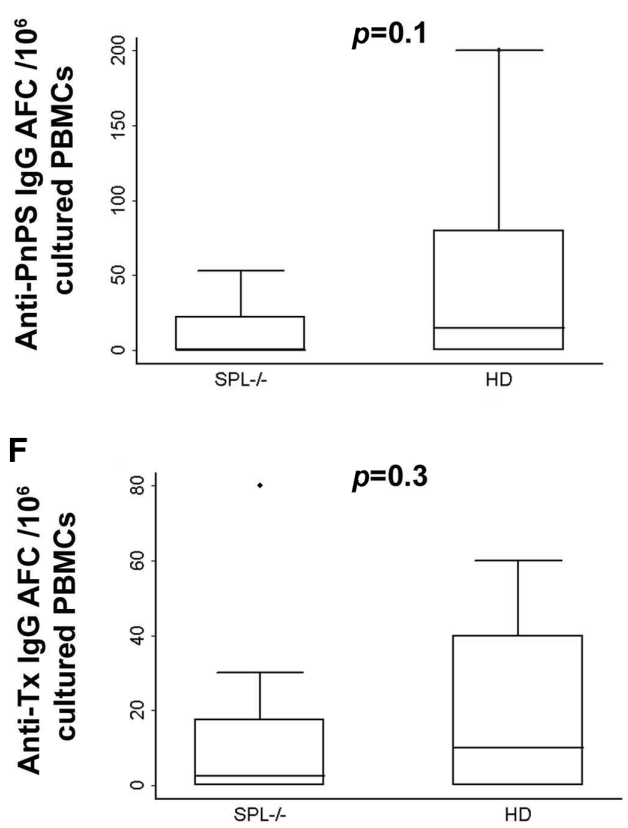

Figure 4. Number of memory B cells secreting IgM, IgG anti-PnPS IgM, and anti-PnPS IgG in the peripheral blood of splenectomized and healthy children. PBMCs were stimulated with CpG for 5 days and the number of antibody secreting cells measured by ELISPOT. (A-F) The number of (A) total IgM, (B) total IgG, (C) anti-PnPS IgM, (D) anti-PnPS IgG, (E) anti-tetanus toxoid (Tx) IgM and (F) anti-Tx IgG per million of total cultured PBMCs in splenectomized and control children are shown. Scales on each graph differ depending on the number of spots detected in each experimental set. Data are expressed as box-and-whisker plots with median (solid line), interquartile ranges (boxes), and minimum and maximum nonoutlier values (whiskers) of 21 asplenic and 19 healthy children. Statistical significance was determined by the Mann-Whitney U test.

used to evaluate vaccine immunogenicity is the level of circulating IgG specific for a given antigen. The World Health Organization has recommended a putative threshold of $\geq 0.35 \mu \mathrm{g} / \mathrm{mL}$ IgG against PnPS as a correlate of protection following conjugate vaccination [24]. The first recommendations for vaccination in asplenic individuals issued by CDC in 1997 are based on the observation that levels of antibodies induced in this group of patients by PnPS vaccine are comparable to those of healthy individuals [25]. Other studies have supported these findings [26,27]. Some authors, though, have shown that response to PnPS vaccination in asplenic individuals can be reduced or absent [28-30], and that antibody titers may decay more rapidly than in healthy individuals $[31,32]$. Thus, the recommendation for revaccination 5 years after the first dose has been formulated [16]. Pneumococcal conjugate vaccines are immunogenic in asplenic individuals [33,34], and recent guidelines $[16,19,35]$ include PCV13 in the vaccination protocol for this group of high-risk patients. Immunization with PnPS vaccine is still recommended, mainly for its greater antigenic repertoire that includes more serotypes as compared to PCV13.

Antibodies represent only one of the products of immunization response. In fact, the germinal center reaction in response to natural infection or vaccination generates two sophisticated tools that have different but complementary protective functions: Long-lived plasma cells and memory B cells [36]. The first, endowed in specialized bone marrow niches, survive virtually forever, they are unable to sense antigen [37], but continuously producing high affinity antibodies that have been selected by previous immunological experiences. Memory B cells, residing preferentially in the spleen, carry antigen-selected antibodies as surface antigen receptors and are therefore able to mount rapid recall responses.

Thus, when a previously encountered pathogen enters the organism, protection is ensured by "ready to use" serum 


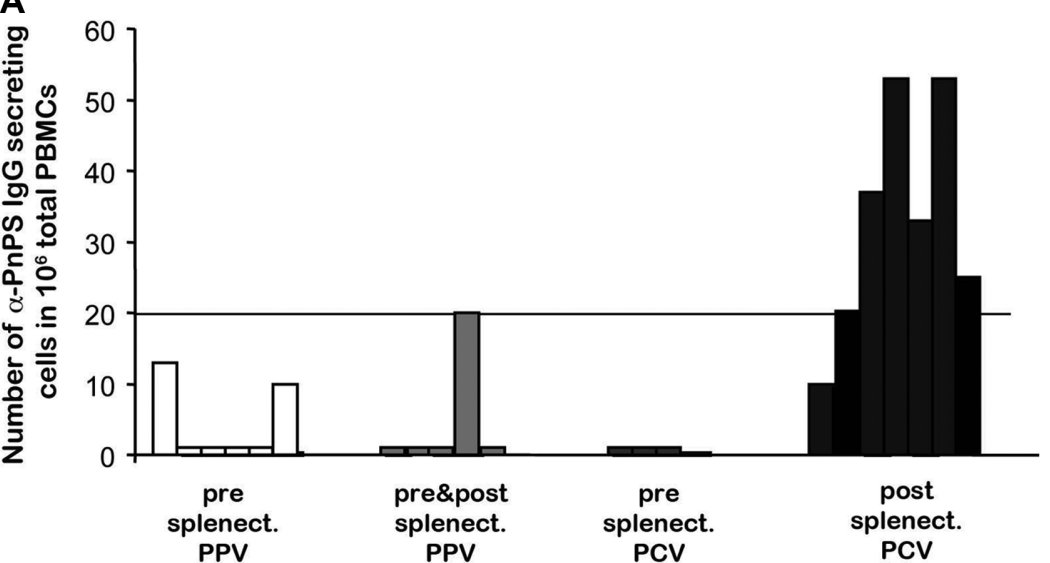

B

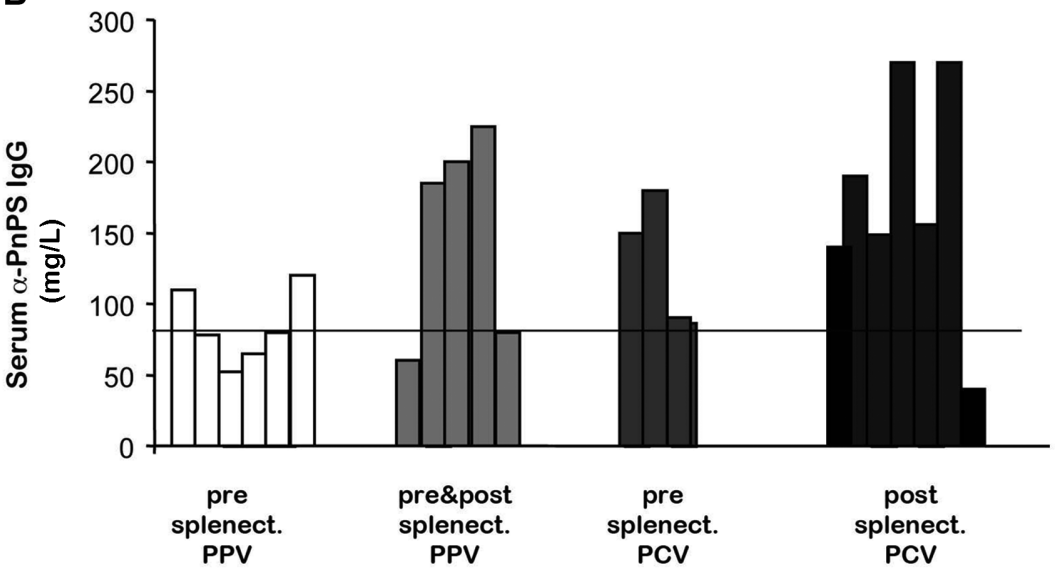

C
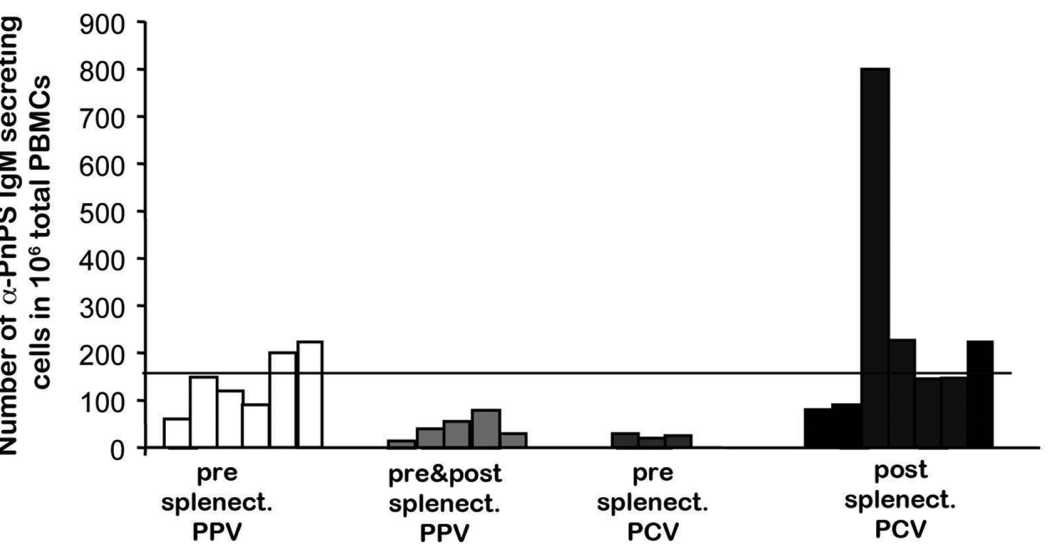

Figure 5. Anti-PnPS IgG serum concentration and number of memory B cells specific for PnPS in splenectomized children grouped according to their vaccination history. (A) The number of anti-PnPS IgG secreting cells per million of total PBMCs in splenectomized children grouped by type of antipneumococcal vaccine received and time of vaccination (before or after splenectomy) is shown. Each bar represents one child. Six children received PnPS vaccination before splenectomy (white bars), five had PPV before and after splenectomy (light gray bars), PCV before splenectomy was administered to three patients (dark gray bars) and to seven patients after splenectomy (black bars). Horizontal line indicates the median value of anti-PnPS IgG secreting cells per million of total PBMCs of healthy controls. (B) The anti-PnPS IgG serum concentrations (mg/L) is shown. Median value for anti-PnPS IgG serum concentration in healthy control is indicated by the horizontal line. (C) The number of anti-PnPS IgM secreting cells per million of total PBMCs in splenectomized children grouped as described above is shown; horizontal line represents the median value of healthy controls. immunoglobulins produced by long-lived plasma cells. Meanwhile, memory B cells bind the antigen, react and in few days, implementing the plasma-cell compartment, increase the level of specific antibodies. It is theoretically difficult to say whether only one of these two branches of protection may be sufficient to avoid disease [38]. We show that splenectomized patients have the same amount of circulating antibodies specific for pneumococcus than healthy individuals of the same age. Based on this finding, additional vaccination should not be necessary.
Our study shows that the difference between individuals that have or lack the spleen is in the frequency of memory B cells. As already known, total memory B cells are reduced after splenectomy. We now demonstrate that lack of this cell population explains both the increased susceptibility to pneumococcal infection and the decreased response to polysaccharide vaccines in asplenic individuals: in fact, according to our findings, $S$. pneumoniae-specific memory B cells are diminished, and remain low in patients that have been vaccinated with pure polysaccharide 
antigens. As confirmed by a recent study [39], T-independent antigens do not generate switched memory B cells and polysaccharide vaccines may even deplete a preexisting pool of specific switched memory B cells [40]. In contrast, it has been shown that PnPS vaccination can induce long-lived plasma cells in the mouse [41]. Thus, the antibodies in the serum of asplenic patients probably derive from long-lived plasma cells either generated by previous natural infections or from vaccination.

The question of whether memory B cells may play a role in the defense against pneumococcal disease can be addressed by considering the case of common variable immunodeficiency. This primary immune deficiency is characterized by a reduced serum level of all immunoglobulin isotypes and a lack of response to vaccination. For most patients, respiratory infections are the main symptoms and may lead to permanent lung damage. A few years ago, a correlation between presence of IgM memory B cells and a decreased incidence of bronchiectasis has been shown in a group of patients that received standard immunoglobulin replacement $[42,43]$. Therefore, it was suggested that IgM memory B cells may be involved in the protection from recurrent pulmonary infections and subsequent lung damage. Nevertheless, it must be pointed out that a tailored immunoglobulin replacement and a correct lifestyle can also protect all common variable immunodeficiency patients from respiratory infections and lung damage [44].

Here, we show that IgM memory B cells able to produce antipneumococcal antibodies are tenfold lower than normal after splenectomy.

We show that, in healthy individuals, the proportion of circulating PnPS-specific IgM memory B-cells over the total number of IgM memory B cells is high (4\%).

This high frequency may be a result of coevolution of our immune system with a ubiquitous pathogen that infects humans from the first days of life. In fact, pneumococci are human commensals, which colonize the nasopharynx of $8-30 \%$ healthy adults and $20-50 \%$ of healthy children under 4 years of age [45]. Therefore, in the absence of any vaccination, many adults already have anti-PnPS IgM and IgG antibodies [46]. Moreover, IgM memory B cells are functionally equivalent to mouse B-1 B cells that produce natural antibodies, elicited by self-antigens or altered selfantigens in complete absence of any antigenic stimulation. These preexisting natural IgM antibodies directly neutralize and inhibit pathogen replication by binding multiple antigens including bacterial polysaccharides [47].

In case of influenza virus infection, pneumococcal carriage increases and, as the local immune response against the virus leads to alterations on the mucosal epithelial layer, bacteria can more easily penetrate the airways and cause infection [48]. In fact, seasonal respiratory syncytial virus (RSV) or influenza epidemics are often associated with pneumonia caused by $S$. pneumoniae [49]. In the particular case of asplenic patients, already at risk of OPSI, annual vaccination against influenza (generating a T-dependent immune response) can be used as an additional tool to protect from bacterial invasive diseases. A recent study has demonstrated that influenza vaccination reduces mortality in splenectomized patients by $54 \%$ [50].
A large proportion of switched memory B cells generated after vaccination resides in the spleen and is lost with its removal [51]. This explains why anti-tetanus IgG memory B cells were significantly reduced in the peripheral blood of asplenic adults. In asplenic children, this difference was not significant, probably because the vaccine is administered at several time points from 2 months to 11 years of life, even after splenectomy. Thus, switched memory B cells specific for tetanus could be produced "de novo" in the germinal centers of the lymph nodes draining the injection site. In contrast, most adults are not revaccinated against tetanus after splenectomy.

Despite the existing recommendation to administer a booster dose of anti-tetanus vaccine every ten years, the compliance to this recommendation is poor. Our present study reinforces the importance of this recommendation, as well as of the need for revaccination against other antigens in order to replenish the pool of switched memory B cells after splenectomy.

Because we have selected our asplenic patients excluding those with immunodeficiencies, autoimmunity, hematologic tumors, or under immunosuppressive therapy, all our observations reflect the "pure" effects of spleen removal. Thus, we can hypothesize that glycoconjugated vaccines may be the most plausible choice for the generation of specific memory B cells after splenectomy.

Our study has some limitations: in our study sample, asplenic children were older than controls. This may have biased our result toward an underestimation of the difference since memory B cells are expected to increase with age. Moreover, the observational design does not allow us to draw definitive conclusions on the efficacy of PCV for generating an appropriate immune response in asplenic individuals.

In conclusion, our study confirms and extends results previously reported by others, shedding light on the crucial role of the spleen in the immunological response to infections caused by encapsulated bacteria. An appropriate prospective clinical trial is needed to better evaluate the efficacy of T-dependent vaccination in splenectomized individuals.

\section{Materials and methods}

\section{Study design and patient recruitment}

Between February 2012 and November 2012, we enrolled a group of asplenic patients and a group of healthy, immunocompetent individuals. Asplenic patients and controls were enrolled in the Hematology Units, Day Surgery and among the personnel of four different Italian hospitals (Bambino Gesù Children's Hospital, Rome; Sapienza University of Rome; Second University of Naples; Policlinico San Matteo, Pavia). Inclusion criteria for asplenic patients were: (1) surgical splenectomy or functional asplenia; (2) detection of Howell-Jolly bodies on blood smears; (3) age above 1 year. Exclusion criteria were: (1) presence of any condition other than asplenia known to impair the immune 
system, including primary and secondary immunodeficiencies, allergies, autoimmune diseases, and therapy with immunosuppressive drugs; (2) unknown immunization history. Controls were selected among healthy children undergoing elective surgery (preputial plasty and other small procedures in Day Surgery) and healthy adult blood donors. Individuals with unknown vaccination history were excluded.

Written, informed consent was obtained. A blood sample $(10 \mathrm{~mL})$ was collected at enrollment. Demographic and epidemiologic data such as birth date, sex, date of splenectomy or of diagnosis of asplenia and cause of the asplenia were collected through a questionnaire filled-up by legal tutors or patients. Vaccine history was obtained from the vaccination record. The study was approved by the Bambino Gesù Children's Hospital's Ethical Committee.

\section{Study population}

We enrolled a total of 144 individuals, 104 adults (57 asplenic and 47 healthy individuals) and 40 children (21 asplenic and 19 healthy children). Asplenic adults had a median age of 35 years (range: 18.2-82.9), and 36 (63\%) were females. As for the healthy adult patients, median age was 31.7 years (range: $21.5-80.5$ ), of them 26 (55\%) were females. Thirty (53\%) of the asplenic adults were affected by thalassemia, 11 (19\%) had been splenectomized for a trauma, $3(5 \%)$ had a diagnosis of spherocytosis, 2 (4\%) had been splenectomized for a nonhematologic neoplasia and 11 (19\%) had been splenectomized for other diseases. Median age at splenectomy was 18.7 years (range 3.3-65.7). Thirty-six of the splenectomized adults (63\%) had received one dose of PnPS vaccine before splenectomy, while the remaining 21 (37\%) had received it after splenectomy. Healthy donors were not vaccinated against pneumococcus.

In the group of patients with age $<18$ years, median age was 13.6 years (range: 1.2-18) and 9 (43\%) were females, whereas the median age of healthy pediatric controls was 7.6 (range: $2.8-16) ; 2$ (10.5\%) were females. One of the asplenic children was affected by congenital asplenia. Among the remaining 20 patients, $16(76 \%)$ had been splenectomized for spherocytosis and 4 (19\%) had undergone posttraumatic splenectomy. Regarding anti-pneumococcal vaccination in the group of asplenic children, six patients had received PnPS vaccination only before splenectomy, five had been immunized with PnPS both before and after splenectomy, three children had been vaccinated with PCV before surgical removal of the spleen, and seven children had received PCV after splenectomy.

\section{Cell isolation and flow-cytometry analysis}

Heparinized PBMCs were isolated by Ficoll Paque ${ }^{\mathrm{TM}}$ Plus (Amersham Pharmacia Biotech) density-gradient centrifugation, counted and used for cell culture (see below) or stained with the appropriate combination of fluorescent-labeled antibodies and analyzed by flow-cytometry [8]. Dead cells were excluded from analysis by side/forward scatter gating. All analyses were performed on a FACSCanto (BD Biosciences) interfaced to PC FACSDiva software. One hundred thousand events per sample were analyzed.

\section{Cell cultures}

For polyclonal stimulation of memory B cells $1-2 \times 10^{6}$ PBMCs were cultured for 5 days in RPMI 1640 (Gibco BRL, Life Technologies) supplemented with $10 \%$ heat inactivated fetal bovine serum (Hyclone Laboratories Logan UT), 2\% L-glutamine (Gibco BRL), $5 \times 10^{-5}$ M 2- $\beta$ mercaptoethanol (Sigma Aldrich, St. Louis, MO) and $20 \mathrm{mg} / \mathrm{mL}$ gentamycin (Gibco BRL), $2.5 \mu \mathrm{g} / \mathrm{mL} \mathrm{CpG-}$ ODN (Hycult Biotechnology, The Netherlands), $20 \mathrm{ng} / \mathrm{mL}$ recombinant IL-21 (Peprotech, UK), and $20 \mathrm{ng} / \mathrm{mL}$ recombinant IL-4 (Peprotech).

\section{ELISPOT}

The 96-well plates (MultiScreen-HA, Milipore) were coated overnight with AffiniPure F(ab')2 Fragment Goat anti-human IgA+IgG+IgM (H+L) (Jackson Immuno Research Laboratories), synthetic tetanus toxin peptide (C-term) (Acris-antibodies, Germany) or PneumoVax ${ }^{\circledR}$ (Sanofi Pasteur MSD) containing S. pneumoniae polysaccharides of the following serotypes $1,2,3,4,5$, $6 \mathrm{~B}, 7 \mathrm{~F}, 8,9 \mathrm{~N}, 9 \mathrm{~V}, 10 \mathrm{~A}, 11 \mathrm{~A}, 12 \mathrm{~F}, 14,15 \mathrm{~B}, 17 \mathrm{~F}, 18 \mathrm{C}, 19 \mathrm{~F}, 19 \mathrm{~A}$, $20,22 \mathrm{~F}, 23 \mathrm{~F}$, and $33 \mathrm{~F}$. After washing with sterile $\mathrm{PBS} / 0.05 \%$ Tween20, plates were blocked for $1 \mathrm{~h}$ at $37^{\circ} \mathrm{C}$ with $\mathrm{PBS} /$ gelatine $1 \%$. PBMCs stimulated for 5 days, as described before, were collected, counted, and seeded in the precoated plates. Plates were left at $37^{\circ} \mathrm{C}, 2 \% \mathrm{CO}_{2}$ for $4-6 \mathrm{~h}$ to allow antibody secretion. The $1 / 2$ serial dilution were done starting in the first well with: $5 \times 10^{4}$ cells for detection of total $\mathrm{IgM}$ and IgG; $2.5 \times$ $10^{5}$ cells for anti-tetanus Igs and $2 \times 10^{5}$ cells for specific antiPnPS Igs. After incubation, plates were washed with $\mathrm{dH}_{2} \mathrm{O} / 0.05 \%$ Tween20 (once) and PBS/0.05\% Tween20 (two times) and incubated overnight with anti-IgM HRPO (1:1000) or anti-IgG HRPO (1:2000) (Jackson Immuno Research Laboratories) diluted in PBS+gelatin $1 \%+0.05 \%$ Tween20 (Sigma). After washing twice as before, TMB substrate (ready to use from Mabtech-ELISpot plus for human IgG kit, Mabtech AB) was used according to the manufacturer's instructions. Plates were left at RT to allow the blue color to develop and the reaction was stopped with $\mathrm{dH}_{2} \mathrm{O}$. Plates were left to dry before counting by two independent blinded operators.

\section{Enzyme-linked immunosorbent assay}

Specific anti-tetanus and anti-PnPS IgG serum concentrations were measured using VaccZyme ${ }^{\mathrm{TM}}$ Anti-Tetanus Toxoid IgG Enzyme Immunoassay kit and VaccZyme ${ }^{\mathrm{TM}}$ Anti-PCP IgG Enzyme Immunoassay kit from Binding Site (The Binding Site), respectively, according to the manufacturer's instructions. For anti-PCP 
IgG concentrations were calculated from the standard curves in $\mathrm{mg} / \mathrm{L}$ and converted in $\mathrm{IU} / \mathrm{mL}(1 \mathrm{IU} / \mathrm{mL}=17 \mathrm{mg} / \mathrm{L})$. Protective IgG serum levels for tetanus are $0.01-0.15 \mathrm{IU} / \mathrm{mL}$.

\section{Spleen samples}

Four samples of splenic tissue were collected from pediatric patients (Supporting Information Table 1) at the moment of splenectomy at the Bambino Gesù Children's Hospital.

\section{Immunohistochemistry}

Immunohistochemistry was performed on formalin-fixed, paraffinembedded spleen tissues using a three-step immunoperoxidase protocol for anti-human CD27 (clone 137B4) (UCS Diagnostics, Italy), rabbit anti-human IgM (DakoCytomation, Denmark), and rabbit anti-human IgG (DakoCytomation) and images acquired using an Axio microscope connected to an Imager A.1 camera (Carl Zeiss, Oberkochen, Germany).

\section{Cell cultures}

Lymphocytes from spleen single cell suspensions were isolated by Ficoll Paque ${ }^{\mathrm{TM}}$ Plus (Amersham Pharmacia Biotech, Uppsala, Sweden) density-gradient centrifugation, counted and used for cell culture. For polyclonal stimulation of memory B cells $1-2 \times 10^{6}$ spleen lymphocytes were cultured for 5 days in RPMI 1640 (Gibco BRL, Life Technologies, Carlsbad, CA) supplemented with 10\% heat inactivated fetal bovine serum (Hyclone Laboratories Logan UT), 2\% L-glutamine (Gibco BRL), $5 \times 10^{-5} \mathrm{M}$ 2 - $\beta$ mercaptoethanol (Sigma, St. Louis) and $20 \mathrm{mg} / \mathrm{mL}$ gentamycin (Gibco BRL), 2,5 mg/mL CpG-ODN (Hycult Biotechnology, Uden, The Netherlands), $20 \mathrm{ng} / \mathrm{mL}$ IL-21 (Peprotech, UK) and $20 \mathrm{ng} / \mathrm{mL}$ IL-4 (Peprotech, UK).

\section{Statistical methods}

The analysis was conducted separately in adults and in children ( $>18$ and $\leq 18$ years). Frequency of B cells, memory B cells, number of total antibody-secreting cells, number of anti-PnPS and anti-Tx IgM and IgG secreting cells, and serum concentration of anti-PnPS and anti-Tx IgG were described through medians, range.

Comparison between immunological values in asplenic patients and healthy individuals was performed through the Mann-Whitney $U$ test. $p$ values lower than 0.05 were considered to be statistically significant.

Acknowledgments: This work has been partly supported by an unrestricted Pfizer grant. M.M.R. was supported by a Mérieux
Research Starting Grant, Institut Merieux, Lyon, France. A special grant $(5 \times 1.000)$ from AIRC (Associazione Italiana Ricerca sul Cancro) also supported the research activity of F.L. We thank Piero De Stefano for editing the manuscript.

Conflict of interest: A.E.T. has received research grants from GlaxoSmithKline and Sanofi Pasteur MSD. All the other authors have no commercial or financial conflict of interest.

\section{References}

1 King, H. and Shumacker, H. B., Jr, Splenic studies. I. Susceptibility to infection after splenectomy performed in infancy. Ann. Surg. 1952. 136: 239-242.

2 Di Sabatino, A., Carsetti, R. and Corazza, G. R., Post-splenectomy and hyposplenic states. Lancet 2011. 378: 86-97.

3 Holdsworth, R. J., Irving, A. D. and Cuschieri, A., Postsplenectomy sepsis and its mortality rate: actual versus perceived risks. Br. J. Surg. 1991. 78: 1031-1038.

4 Red book. In Pickering, L. K. (Ed.), Immunocompromised children (29 ${ }^{\text {th }}$ ed). American Academy of Pediatrics, Elk Grove Village, IL, 2012, pp. 74-90.

5 Mebius, R. E. and Kraal, G., Structure and function of the spleen. Nat. Rev. Immunol. 2005. 5: 606-616.

6 Kang, Y. S., Kim, J. Y., Bruening, S. A., Pack, M., Charalambous, A., Pritsker, A., Moran, T. M. et al., The C-type lectin SIGN-R1 mediates uptake of the capsular polysaccharide of streptococcus pneumoniae in the marginal zone of mouse spleen. Proc. Natl. Acad. Sci. USA 2004. 101: 215-220.

7 Foote, J. B., Mahmoud, T. I., Vale, A. M. and Kearney, J. F., Long-term maintenance of polysaccharide-specific antibodies by IgM-secreting cells. J. Immunol. 2012. 188: 57-67.

8 Kruetzmann, S., Rosado, M. M., Weber, H., Germing, U., Tournilhac, O., Peter, H. H., Berner, R. et al., Human immunoglobulin M memory B cells controlling streptococcus pneumoniae infections are generated in the spleen. J. Exp. Med. 2003. 197: 939-945.

9 Weller, S., Faili, A., Garcia, C., Braun, M. C., Le Deist, F. F., de Saint Basile, G. G., Hermine, O. et al., CD40-CD40L independent Ig gene hypermutation suggests a second B cell diversification pathway in humans. Proc. Natl. Acad. Sci. USA 2001. 98: 1166-1170.

10 Weller, S., Braun, M. C., Tan, B. K., Rosenwald, A., Cordier, C., Conley, M. E., Plebani, A. et al., Human blood IgM "memory" B cells are circulating splenic marginal zone B cells harboring a prediversified immunoglobulin repertoire. Blood 2004. 104: 3647-3654.

11 Capolunghi, F., Cascioli, S., Giorda, E., Rosado, M. M., Plebani, A., Auriti, C., Seganti, G. et al., CpG drives human transitional B cells to terminal differentiation and production of natural antibodies. J. Immunol. 2008. 180: $800-808$.

12 Liu, Y. J., Oldfield, S. and MacLennan, I. C., Memory B cells in T celldependent antibody responses colonize the splenic marginal zones. Eur. J. Immunol. 1988. 18: 355-362.

13 Steiniger, B., Timphus, E. M., Jacob, R. and Barth, P. J., CD27+ B cells in human lymphatic organs: re-evaluating the splenic marginal zone. Immunology 2005. 116: 429-442.

14 Coignard-Biehler, H., Lanternier, F., Hot, A., Salmon, D., Berger, A., de Montalembert, M., Suarez, F. et al., Adherence to preventive measures after splenectomy in the hospital setting and in the community. J. Infect. Public. Health 2011. 4: 187-194. 
15 Cadili, A. and de Gara, C., Complications of splenectomy. Am. J. Med. 2008. 121: 371-375.

16 Centers for Disease Control and Prevention (CDC) and Advisory Committee on Immunization Practices, Updated recommendations for prevention of invasive pneumococcal disease among adults using the 23-valent pneumococcal polysaccharide vaccine (PPSV23). MMWR Morb. Mortal. Wkly. Rep. 2010. 59: 1102-1106.

17 Centers for Disease Control and Prevention (CDC), Use of 13-valent pneumococcal conjugate vaccine and 23-valent pneumococcal polysaccharide vaccine for adults with immunocompromising conditions: recommendations of the advisory committee on immunization practices (ACIP). MMWR Morb. Mortal. Wkly. Rep. 2012. 61: 816-819.

18 Pickering, L., Baker, C., Kimberlin, D. and Long, S., Red book: 2012 report of the committee on infectious diseases. Am. Acad. Pediatr. 2012. 29: 11058.

19 Nuorti, J. P., Whitney, C. G. and Centers for Disease Control and Prevention (CDC), Prevention of pneumococcal disease among infants and children-use of 13-valent pneumococcal conjugate vaccine and 23valent pneumococcal polysaccharide vaccine-recommendations of the advisory committee on immunization practices (ACIP). MMWR Recomm. Rep. 2010. 59: 1-18.

20 Reinecke, G. and Pabst, R., Subsets of blood, spleen and recirculating lymphocytes in man. Clin. Exp. Immunol. 1983. 53: 672-678.

21 Carsetti, R., Rosado, M. M. and Wardmann, H., Peripheral development of B cells in mouse and man. Immunol. Rev. 2004. 197: 179-191.

22 Rosado, M. M., Scarsella, M., Pandolfi, E., Cascioli, S., Giorda, E., Chionne, P., Madonne, E. et al., Switched memory B cells maintain specific memory independently of serum antibodies: the hepatitis B example. Eur. J. Immunol. 2011. 41: 1800-1808.

23 Janeway, C. A., Travers, P., Walport, M. and Capra, J. D., Immunobiology: The Immune System in Health and Disease. Current Biology, Garland Science, New York, 2001.

24 World Health Organization, Recommendations for the production and control of pneumococcal conjugate vaccines. WHO Tech. Rep. Ser. 2005. 927: 64-98.

25 Ammann, A. J., Addiego, J., Wara, D. W., Lubin, B., Smith, W. B. and Mentzer, W. C., Polyvalent pneumococcal-polysaccharide immunization of patients with sickle-cell anemia and patients with splenectomy. N. Engl. J. Med. 1977. 297: 897-900.

26 Landgren, O., Bjorkholm, M., Konradsen, H. B., Soderqvist, M., Nilsson, B., Gustavsson, A., Axdorph, U. et al., A prospective study on antibody response to repeated vaccinations with pneumococcal capsular polysaccharide in splenectomized individuals with special reference to hodgkin's lymphoma. J. Intern. Med. 2004. 255: 664-673.

27 Stanford, E., Print, F., Falconer, M., Lamden, K., Ghebrehewet, S., Phin, N., Baxter, D. et al., Immune response to pneumococcal conjugate vaccination in asplenic individuals. Hum. Vaccin. 2009. 5: 85-91.

28 Musher, D. M., Ceasar, H., Kojic, E. M., Musher, B. L., Gathe, J. C., Jr, Romero-Steiner, S. and White, A. C., Jr, Administration of proteinconjugate pneumococcal vaccine to patients who have invasive disease after splenectomy despite their having received 23-valent pneumococcal polysaccharide vaccine. J. Infect. Dis. 2005. 191: 1063-1067.

29 Cherif, H., Landgren, O., Konradsen, H. B., Kalin, M. and Bjorkholm, M., Poor antibody response to pneumococcal polysaccharide vaccination suggests increased susceptibility to pneumococcal infection in splenectomized patients with hematological diseases. Vaccine 2006. 24: 75-81.

30 Llupia, A., Vilella, A., Costas, L., Diez, C., Torres, F., Yague, J., Masso, M. et al., Can the response to 23-valent pneumococcal vaccine in splenectomised patients be predicted? Vaccine 2012. 30: 2382-2386.
31 Giebink, G. S., Le, C. T. and Schiffman, G., Decline of serum antibody in splenectomized children after vaccination with pneumococcal capsular polysaccharides. J. Pediatr. 1984. 105: 576-582.

32 Mikoluc, B., Kayhty, H., Bernatowska, E. and Motkowski, R., Immune response to the 7-valent pneumococcal conjugate vaccine in 30 asplenic children. Eur. J. Clin. Microbiol. Infect. Dis. 2008. 27: 923-928.

33 Smets, F., Bourgois, A., Vermylen, C., Brichard, B., Slacmuylders, P., Leyman, S. and Sokal, E., Randomised revaccination with pneumococcal polysaccharide or conjugate vaccine in asplenic children previously vaccinated with polysaccharide vaccine. Vaccine 2007. 25: 5278-5282.

34 Mikoluc, B., Kayhty, H., Bernatowska, E. and Motkowski, R., Immune response to the 7-valent pneumococcal conjugate vaccine in 30 asplenic children. Eur. J. Clin. Microbiol. Infect. Dis. 2008. 27: 923-928.

35 Davies, J. M., Lewis, M. P., Wimperis, J., Rafi, I., Ladhani, S., BoltonMaggs, P. H. and British Committee for Standards in Haematology, Review of guidelines for the prevention and treatment of infection in patients with an absent or dysfunctional spleen: prepared on behalf of the British committee for standards in haematology by a working party of the haemato-oncology task force. Br. J. Haematol. 2011. 155: 308-317.

36 Shlomchik, M. J. and Weisel, F., Germinal center selection and the development of memory B and plasma cells. Immunol. Rev. 2012. 247: 52-63.

37 Manz, R. A., Cassese, G., Thiel, A. and Radbruch, A., Long-lived plasma cells survive independent of antigen. Curr. Top. Microbiol. Immunol. 1999. 246: 71-74; discussion 74-75.

38 Pichichero, M. E., Booster vaccinations: can immunologic memory outpace disease pathogenesis? Pediatrics 2009. 124: 1633-1641.

39 Truck, J., Lazarus, R., Jonsdottir, I., Klugman, K. P. and Pollard, A. J., Pneumococcal polysaccharide vaccine efficacy and routine use of conjugate vaccines in infants: there is no need for a vaccine program in older adults at present. Clin. Infect. Dis. 2012. 55: 1577-1579; author reply 1579-1581.

40 Clutterbuck, E. A., Lazarus, R., Yu, L. M., Bowman, J., Bateman, E. A., Diggle, L., Angus, B. et al., Pneumococcal conjugate and plain polysaccharide vaccines have divergent effects on antigen-specific B cells. J. Infect. Dis. 2012. 205: 1408-1416.

41 Taillardet, M., Haffar, G., Mondiere, P., Asensio, M. J., Gheit, H., Burdin, N., Defrance, T. and Genestier, L., The thymus-independent immunity conferred by a pneumococcal polysaccharide is mediated by long-lived plasma cells. Blood 2009. 114: 4432-4440.

42 Capolunghi, F., Rosado, M. M., Sinibaldi, M., Aranburu, A. and Carsetti, R., Why do we need IgM memory B cells? Immunol. Lett. 2013. 152: 114-120.

43 Carsetti, R., Rosado, M. M., Donnanno, S., Guazzi, V., Soresina, A., Meini, A., Plebani, A. et al., The loss of IgM memory B cells correlates with clinical disease in common variable immunodeficiency. J. Allergy Clin. Immunol. 2005. 115: 412-417.

44 Lucas, M., Lee, M., Lortan, J., Lopez-Granados, E., Misbah, S. and Chapel, H., Infection outcomes in patients with common variable immunodeficiency disorders: relationship to immunoglobulin therapy over 22 years. J. Allergy Clin. Immunol. 2010. 125: 1354-1360.e4.

45 Hussain, M., Melegaro, A., Pebody, R. G., George, R., Edmunds, W. J., Talukdar, R., Martin, S. A. et al., A longitudinal household study of streptococcus pneumoniae nasopharyngeal carriage in a UK setting. Epidemiol. Infect. 2005. 133: 891-898.

46 Goldblatt, D., Hussain, M., Andrews, N., Ashton, L., Virta, C., Melegaro, A., Pebody, R. et al., Antibody responses to nasopharyngeal carriage of streptococcus pneumoniae in adults: a longitudinal household study. J. Infect. Dis. 2005. 192: 387-393. 
47 Baumgarth, N., The double life of a B-1 cell: self-reactivity selects for protective effector functions. Nat. Rev. Immunol. 2011. 11: 34-46.

48 McCullers, J. A., Insights into the interaction between influenza virus and pneumococcus. Clin. Microbiol. Rev. 2006. 19: 571-582.

49 Rice, T. W., Rubinson, L., Uyeki, T. M., Vaughn, F. L., John, B. B., Miller, R. R., 3rd, Higgs, E. et al., Critical illness from 2009 pandemic influenza A virus and bacterial coinfection in the United States. Crit. Care Med. 2012. 40: $1487-1498$

50 Langley, J. M., Dodds, L., Fell, D. and Langley, G. R., Pneumococcal and influenza immunization in asplenic persons: a retrospective populationbased cohort study 1990-2002. BMC Infect. Dis. 2010. 10: 219-2334-10-219.

51 Mamani-Matsuda, M., Cosma, A., Weller, S., Faili, A., Staib, C., Garcon, L., Hermine, O. et al., The human spleen is a major reservoir for longlived vaccinia virus-specific memory B cells. Blood 2008. 111: 4653-4659.
Abbreviations: OPSI: overwhelming postsplenectomy infection - PCV: pneumococcal-conjugated vaccine - PBMC: peripheral blood mononuclear cell · PnPS: pneumococcal polysaccharide

Full correspondence: Dr. M. Manuela Rosado, Unit of B-cell development, Bambino Gesù Children's Hospital (IRCCS), Piazza S. Onofrio 4, 00165 Rome, Italy Fax: +39-0668592904

e-mail:m.rosado@wildmail.com

Received: $28 / 3 / 2013$

Revised: 17/6/2013

Accepted: 25/6/2013

Accepted article online: 28/6/2013 